\title{
DOCUMENTS
}

Hans-Josef Steinberg

\section{FREIHEIT UND NOTWENDIGKEIT: AUS EINEM VERLORENEN BRIEF VON FRIEDRICH ENGELS AN ERNEST BELFORT BAX VOM JAHRE 1886}

Ende März, Anfang April 1899 polemisierte Karl Kautsky in der Neuen Zeit gegen die einige Wochen zuvor erschienene Broschüre Eduard Bernsteins Die Voraussetzungen des Sozialismus und die Aufgaben der Sozialdemokratie. ${ }^{1}$ Bernstein eröffnete die Erwiderung mit dem Artikel „Die Notwendigkeit in Natur und Geschichte”.2 Im Zusammenhang mit der Frage nach dem Determinismus im historischen Materialismus verwies er in einer Fußnote auf einen Engels-Brief. Die Anmerkung lautet: „Nach Kautsky (,Neue Zeit', Nr. 27, S. 7) ist der Determinismus der ,tragende Pfeiler' der materialistischen Geschichtsauffassung. Welcher Determinismus? In einem Briefe aus dem Jahre 1886, den mir nach Drucklegung des obigen Artikels der Zufall unter die Augen geführt hat, erklärt sich Engels ausdrücklich als Gegner des strengen Determinismus. Ich habe nicht das Recht, den Brief, der anvertrautes Gut ist, jetzt zu veröffentlichen, indes braucht man nur die Stelle über Notwendigkeit und Freiheit im Anti-Dühring genauer nachzulesen, um sich zu überzeugen, daß Engels dem materialistischen Determinismus durchaus ketzerisch gegenüberstand."3

Am 17.5.1899 schrieb darauf Kautsky an Bernstein: „Aus einer Fußnote Deines Artikels erfahre ich, daB Du Dich für den Determinismus auf einen noch unveröffentlichten Brief Generals berufst. Das legt mir den Wunsch nahe, auch in denen, die er mir gesandt hat, nach Äußerungen darüber zu suchen. Ich habe Dir s[einer] $Z[$ eit] diese Briefe übergeben. Bitte, schicke sie mir eingeschrieben zurück. Mir sind sie schon einigemale sehr abgegangen. Sollte die Stelle, auf die Du Dich berufst, etwa in einem Brief an mich enthalten sein, dann erteile ich Dir von vornherein das Recht, sie zu publizieren.

1 Vgl. K. Kautsky, „Bernstein und die materialistische Geschichtsauffassung”; „Bernstein und die Dialektik”; „Bernstein über die Werttheorie und die Klassen", in: Die Neue Zeit, XVII, 2 (1899), S. 4-16, 36-50, 68-81.

2 Ebenda, S. 260-269.

3 Ebenda, S. 266. 
Aber umso mehr wünschte ich dann, den ganzen Brief samt früheren und späteren wieder lesen zu können." 1 Gereizt antwortete Bernstein, ${ }^{2}$ und Kautsky versuchte zwar, ihn zu beschwichtigen, bestand aber weiterhin darauf, genauer über den Inhalt des Engels-Briefes informiert zu werden. ${ }^{3}$ In einem längeren Schreiben vom 6. Juni 1899 teilte Bernstein Kautsky die hinsichtlich des zur Debatte stehenden Themas wesentlichen Passagen aus dem besagten Engels-Brief mit. Das Original des Schreibens von Engels gilt als verloren, und es ist ein ausgesprochener Glücksfall, daß die überaus wesentlichen Aussagen, die Engels darin über Freiheit und Notwendigkeit macht, durch die längeren zusammenhängenden Zitate im Brief Bernsteins an Kautsky überliefert worden sind.

Niemand wird behaupten wollen, daß die im folgenden zitierten Äußerungen von Engels für den Revisionismus Bernsteinscher Provenienz vereinnahmt werden können, und so schrieb auch schon Kautsky am 29.6.1899 an Bernstein: „Ich deute diese Stelle ganz anders als Du und kann ihr vollkommen zustimmen." 4 In der Tat handelt es sich hier um eine kurze und präzise Fassung dessen, was Friedrich Engels im selben Jahr in Ludwig Feuerbach und der Ausgang der klassischen deutschen Philosophie über die wesentlich verschiedenartige Entwicklungsgeschichte von Gesellschaft und Natur ausgeführt hat. ${ }^{5}$ Damit ist ein weiterer Beleg dafür gegeben, daB das Verhältnis von Freiheit und Notwendigkeit im Marxismus durch kurzschlüssige Interpretationen der Engelschen Aussage im Anti-Dühring, nach der die Freiheit die Einsicht in die Notwendigkeit sei, ${ }^{6}$ wobei die Erläuterungen, die Engels dazu gibt, übergangen werden, nicht zureichend erfaßt werden kann.

Im folgenden wird der gesamte Brief Bernsteins an Kautsky vom 6.6.1899 abgedruckt. Die Zitate aus dem verlorenen Schreiben von Engels werden in Kursivdruck wiedergegeben. Die Schreibweise wurde

1 Kautsky an Bernstein, 17.5.1899, IISG, Kautsky-Nachlaß, C 225.

${ }^{2}$,Deine Engelsbriefe gehen morgen in einem Paket an Dich ab. Wie Du sehen wirst, befinden sie sich in demselben Zustand, in dem Du sie mir s[einer] Z[ei]t anvertraut hast. Ob ein Brief über Determinismus darunter ist, weiß ich daher nicht. Jedenfalls entspricht es nicht meinen Gewohnheiten, einen Brief, den Engels an Dich geschrieben, in solcher Weise gegen Dich auszuspielen. Daß Du so etwas annehmen konntest, erklärt mir die Zurückforderung der Briefe und noch manches andere." (Bernstein an Kautsky, 20.5.1899, ebenda, DV 478)

3 Kautsky an Bernstein, 24.5.1899: „Was ich unangenehm empfinde, ist bloB der Umstand, daß Du nicht den Wortlaut der Stelle mitteilst, uns also ihre Prüfung unmöglich machst" (ebenda, C 226).

4 IISG, Kautsky-NachlaB, C 229.

5 Vgl. MEW, Bd 21, S. 296ff. Hierzu auch Engels an W. Borgius, 25.1.1894, und Engels an Joseph Bloch, 21.9.1890, MEW, Bd 39, S. 205-207, Bd 37, S. 462-465.

- MEW, Bd 20, S. $106 f$. 
- wie auch bei den anderen hier zitierten unveröffentlichten Briefen modernisiert.

146 Hithergreen Lane

S. E. London 6. Juni 1899

Lieber Baron,

Endlich ist nun auch der SchluB meiner Antwort auf Deinen Artikel fertig und an Dich abgeschickt. $\mathrm{Zu}$ meinem Bedauern sind es wieder zwei Artikel geworden: einer über die Werttheorie und ein zweiter über die Klassen und den Klassenkampf. ${ }^{1}$ Es ging nicht gut, die beiden Themata in einem abzumachen. Nachdem Du mich herausgefordert, mußte ich auf die Werttheorie etwas näher eingehen. Bei alledem habe ich mich möglichst kurz gefaßt, wie denn überhaupt die große Schwierigkeit dieser ganzen Artikel das Problem war, sich zu konzentrieren, ohne mir den Vorwurf des Ausweichens zuzuziehen, mit dem Du ja nicht sparsam bist.

Wenn ich eine Andeutung von Dietz ${ }^{2}$ recht verstehe, bist Du dabei, ein Buch gegen mich zu schreiben. ${ }^{3}$ Damit wird wohl unsere Polemik in der „Neuen Zeit” ein Ende haben.

Nach der Antwort, die ich am Schluß des Klassenartikels auf Deine Alternative: „konsequenter Marxist oder Kathedersozialist” gebe, wirst Du nun wohl auch Deine endgültige Entscheidung über mein Verbleiben bei der "Neuen Zeit” fällen können. Warum ich Dir die Entscheidung nicht abnehmen kann, habe ich Dir schon dargelegt. ${ }^{5}$ Von Dietz, den ich jüngst fragte, wie es mit der Sache stünde, erhielt ich nur die Antwort, daß ich von ihm keine Kündigung des Verhältnisses zur „Neuen Zeit” zu erwarten habe.

Und zu Deinem Brief betr. der Engels-Briefe. Deine Erklärung, daß die Zurückforderung kein Mißtrauensbeweis sein sollte, akzeptiere ich natürlich. Daß ich sie so auffassen mußte, wirst $\mathrm{Du}$ aber be-

"Vgl. Eduard Bernstein, „Arbeitswert oder Nutzwert?”, in: Die Neue Zeit, XVII, 2 (1899), S. 548-554; „Klassenkampf-Dogma und Klassenkampf-Wirklichkeit", ebenda, S. 577-584, 619-626.

2 Johann Heinrich Wilhelm Dietz (1843-1922), Verleger der "Neuen Zeit”.

${ }^{3}$ Gemeint ist Karl Kautsky, Bernstein und das sozialdemokratische Programm. Eine Antikritik, Stuttgart 1899.

Vgl. Karl Kautsky, „Bernstein über die Werttheorie und die Klassen”, a.a.O., S. 81 .

5 Der Briefwechsel zwischen Kautsky und Bernstein zeigt, daß Kautsky vergeblich versucht hat, Bernstein zum freiwilligen Rücktritt als ständiger Mitarbeiter der „Neuen Zeit" zu bewegen. Bernsteins Gegenargumentation: Kautsky müsse entscheiden, ob ein Mann wie Bernstein noch tragbar für die „Neue Zeit" sei. 
greifen, wenn Du alle die begleitenden Umstände in Betracht ziehst. In Deinen Artikeln hattest Du mich nicht nur als Abgefallenen hingestellt, sondern auch Wendungen gebraucht, die mich als quasi Fälscher an Engels erscheinen ließen. Dann kam Laura Lafargue und forderte die Marx-Engels-Manuskripte betreffs Bruno Bauer von mir heraus, und ein Zweifel, woher dies Geschoß kam, ist nach Lage der Dinge ausgeschlossen. Ferner bleibt einem ja doch nicht vorenthalten, wie Du Dich brieflich über mich äußerst, und nun kommst Du und verlangst die Briefe von Engels von mir zurück, wobei Du noch der Vermutung Ausdruck gibst, als sei der Brief, den ich polemisch gegen Dich erwähne, ein von Engels an Dich gerichteter Brief. Versetzest Du Dich in meine Lage, dann wirst Du begreifen, wieso ich $\mathrm{zu}$ der Auffassung kam, hinter der Rückforderung der Briefe stecke etwas mehr als bloß der Wunsch, sie nachträglich wieder durchzulesen. Ganz offen bist $\mathrm{Du}$ ja auch tatsächlich mir gegenüber jetzt nicht mehr.

Der Brief von Engels, auf den ich Bezug nehme, ist an Bax gerichtet. Bax brachte mir ihn eines Tages, und ich legte ihn zu Briefen, die ein Klubmitglied ${ }^{2}$ Julius $^{3}$ übergeben hatte. Dieses Mitglied forderte nun neulich die Briefe zurück (beziehentlich die Person, von der er sie hatte), und als ich sie herausnahm, fiel mein Blick auf den Baxbrief und zufällig auf die Worte: „b) as to freedom and necessity." Nun blickte ich natürlich näher zu und las weiter: "a I think you do not give $H$. ${ }^{\mathrm{b}}$ his due, but that is because you are yourself such a strict determinist." Engels gibt dann Hegels Standpunkt wieder und fährt fort: „And if you say that our consciousness but registers a foregone necessity, you overlook, in my opinion, that consciousness may register, but that the will acts and reacts upon the outer world, and that this reaction, - necessary though it be every bit - gives to our conscious necessary action quite a different stamp as compared with the unconscious and unwilled necessary action of the other natural agents. I am quite willing to drop the word freedom if you can find a more correct and less equivocal term which at the same time expresses that essential difference."

Die Anführung fehlt im Bernstein-Brief

b Bernstein hat hier ein Kreuz eingefügt und vermerkt am Rand des Briefes +Hegel

1 Ernest Belfort Bax (1854-1926), englischer Sozialist, seit 1883 mit Engels befreundet; 1896-98 kam es zu einer heftigen Diskussion über den Historischen Materialismus zwischen Bax auf der einen und Kautsky und Bernstein auf der anderen Seite. Vgl. hierzu die entsprechenden Jahrgänge der „Neuen Zeit”.

3 Mitglied des Kommunistischen Arbeiterbildungsvereins in London.

3ulius Motteler (1838-1907). Er war unter dem Sozialistengesetz als "Roter Feldpostmeister" berühmt geworden. Nach seiner Ausweisung aus der Schweiz (1888) lebte er bis 1901 in London. 
Die Unterstreichungen sind die Engelsschen im Briefe.

Ich habe keine große Neigung, Bax um die Erlaubnis anzugehen, den Brief stückweise zu publizieren, denn ich stehe mit ihm außer jedem Verkehr. Daß er über Deine Beurteilung meines Buches beglückt ist, steht natürlich außer Frage. Noch habe ich Dir mitzuteilen, daß mich ein Landsmann von Dir, Fr. O. Hertz, der ein Buch über die Agrarfrage geschrieben hat, ${ }^{1}$ mich ersucht hat, dem Buch einige Begleitzeilen auf den Weg zu geben. Da sein Standpunkt dem meinigen sehr verwandt ist und die Arbeit jedenfalls von sehr viel Fleiß und Sachkunde Zeugnis gibt, habe ich ihm zugesagt. Das einzige für mich Störende dabei ist, daB H[ertz] ein paarmal lebhaft gegen Dich Sturm läuft. Das gibt der Sache einen persönlichen Anstrich, denn vor der Welt stehen wir ja jetzt als Gegner da.

Richtig, noch eines. Du scheinst nicht zu wissen, daß die ganzen Engelsschen Briefschaften noch ununtersucht in einem Koffer liegen, von dem August den einen und ich den andern Schlüssel habe. ${ }^{2}$ Dort mag auch der Brief bzw. das Manuskript des unterdrückten Schlusses vom Vorwort zu den Klassenkämpfen sich befinden. ${ }^{3}$ Ich habe nur die Manuskripte und vereinzelte mir nach Engels' Tod zugesandte Briefe an Dritte. Mit herzlichen Grüßen von Gina und mir an Dich und Luise

Dein Ede

1 Vgl. Friedrich O. Hertz, Die agrarischen Fragen im Verhältnis zum Sozialismus. Mit einer Vorrede von Ed. Bernstein, Wien 1899.

2 Engels hatte in seinem Testament seine Manuskripte und Briefe Bebel und Bernstein vermacht. Vgl. MEW, Bd 39, S. 506, 510.

${ }^{3}$ Vgl. hierzu Hans-Josef Steinberg, „Revolution und Legalität. Ein unveröffentlichter Brief Friedrich Engels' an Richard Fischer", in : International Review of Social History, XII (1967), S. 177-189. 